\title{
Lessons from Influenza Outbreaks for Potential Impact of COVID-19 Outbreak on Hospitalizations, Ventilator Use, and Mortality Among Homeless Persons in New York State
}

$\mathrm{J}$ Gen Intern Med 35(9):2781-3

DOI: $10.1007 / \mathrm{s} 11606-020-05876-1$

(C) Society of General Internal Medicine 2020

\section{INTRODUCTION}

Increasing numbers of patients infected by COVID-19 is raising serious concerns about the potential shortage of hospital beds and ventilators in cities such as New York City. The homeless population is particularly large in urban areas and will become larger given the economic crisis. ${ }^{1,2}$ They are high-risk of contagious diseases ${ }^{3}$ (as shelters and drop-in centers are crowded with an active turnover of people), and the outbreak among this population would negatively impact the health care system's ability to respond to this crisis. However, little is understood how the outbreak of infectious diseases among the homeless population affects health systems' resources (e.g., hospital beds, ventilators) and availability of resources for non-homeless population. To address this knowledge gap, using influenza outbreaks as an example, we investigated health care use among the homeless population hospitalized in New York State.

\section{METHODS}

We used the 2007-2012 New York State Inpatient Database that includes all inpatient discharge records from acute care hospitals. We identified all hospitalizations with primary or secondary diagnosis of influenza $(I C D$ 9- $C M$ code 487.xx or 488.xx) from July 2007 to June 2012 (median age 40 years [IQR 6-66]). Homeless patients were identified from the homeless status indicator, which was directly reported by hospitals. ${ }^{4}$

We calculated monthly trends of hospitalization rates (/1000 person-months) in the homeless and nonhomeless populations. Population estimates (denominator) were derived from the US Department of Housing and Urban Development Continuum of Care data for the homeless population, ${ }^{1}$ and the US Census Bureau data for the non-homeless population. ${ }^{5}$ Next, we compared the utilization of care and patient outcomes (hospitalization through emergency department [ED],

Received April 7, 2020

Accepted April 28, 2020

Published online June 4, 2020 mechanical ventilation use [non-invasive or invasive], and in-hospital death) between homeless versus nonhomeless patients using multivariable modified Poisson regression models. We adjusted for patient characteristics and year-month fixed effects. The study was approved by the UCLA Institutional Review Board.

\section{RESULTS}

Of the 20,078 patients hospitalized for influenza across 214 hospitals in New York, 1295 (6.4\%) were homeless patients. Most hospitalizations (99.9\%) among homeless individuals were concentrated in ten hospitals. Homeless patients experienced a higher rate of hospitalization for influenza than non-homeless persons throughout the observation period (Fig. 1). The difference was particularly salient for the pandemic of H1N1 influenza in 2009: hospitalization rates were 2.9 per 1000 for homeless versus 0.1 per 1000 for non-homeless populations. After adjusting for potential confounders, homeless patients were more likely to be hospitalized from ED (adjusted rate ratio $[\mathrm{aRR}], 1.09 ; 95 \%$ CI $1.04-1.14 ; P<0.001)$ and receive mechanical ventilation $(\mathrm{aRR}, 1.58 ; 95 \% \mathrm{CI}$ $1.03-2.43 ; P=0.04)$, compared with non-homeless patients (Table 1). We found no evidence that the inhospital mortality rate differed between homeless and non-homeless populations.

\section{DISCUSSION}

Using the population-based data, including all hospitalizations for influenza in New York, we found that homeless persons had higher utilization of care compared with non-homeless persons. Homeless people experienced a higher hospitalization rate, particularly during a pandemic period and, once hospitalized, a higher rate of mechanical ventilation use. In the midst of COVID-19 outbreak, these findings underscore the importance of infection control and prevention of COVID19 among the homeless population (e.g., lower threshold of virus testing, providing temporary housing to improve sanitation, and social/physical distancing ${ }^{6}$ ), which will, in turn, save resources of the US health care system for larger populations. 


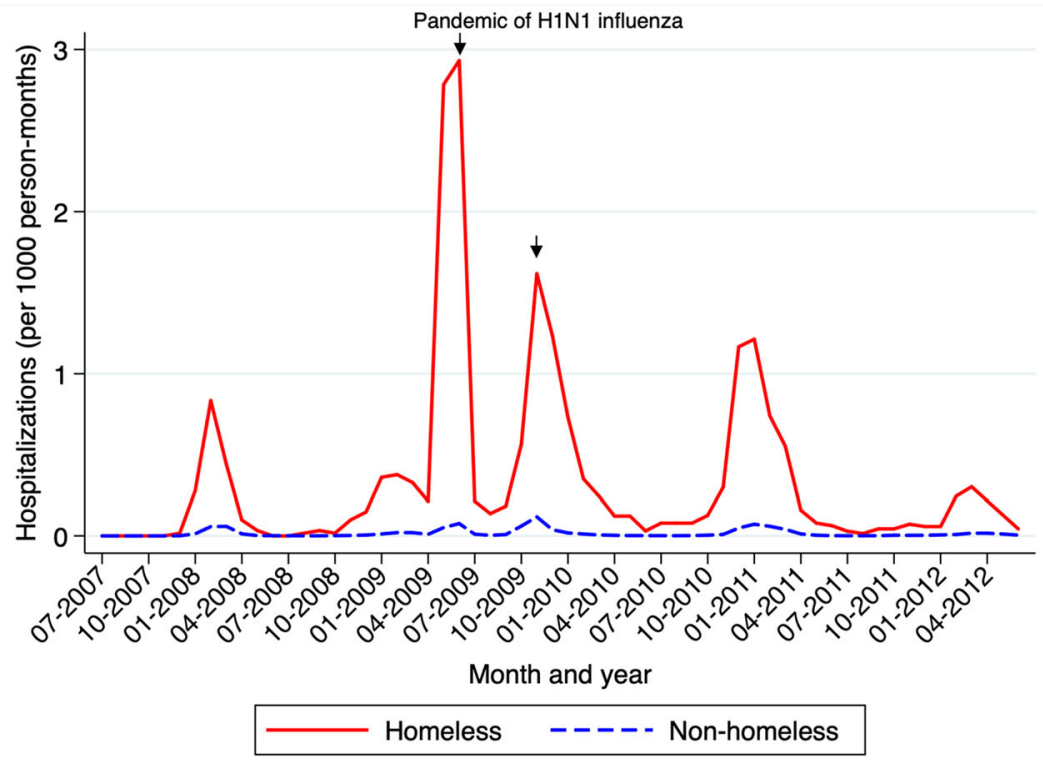

Figure 1 Monthly trend in influenza hospitalizations per 1000 homeless and non-homeless individuals in New York state from July 2007 to June 2012. The arrows indicate the 2009-2010 pandemic of H1N1 influenza. Population estimates for homeless persons were derived from the US Department of Housing and Urban Development 2007-2012 Continuum of Care: Homeless Populations and Subpopulations reports, which provide summary information on point-in-time unduplicated counts of homeless individuals. Corresponding population estimates for nonhomeless persons were derived from using 2007-2012 census data from the US Census Bureau.

Our study has limitations. First, it is possible that homeless indicators were under-coded; therefore, their hospitalization rates might have been underestimated. Second, our findings may not be generalizable to other states than New York. However, New York is currently struck by the outbreak of COVID-19 infections. Furthermore, our data represented $16 \%$ of the entire US homeless population, ${ }^{1}$ and the underlying patterns may be similar across states.

Table 1 Association Between Homelessness and Health Care Indicators in Patients Hospitalized for Influenza

\begin{tabular}{|c|c|c|c|c|c|}
\hline & Outcome, $n$ & Crude rate $(\%)$ & Adjusted rate ratio & $95 \% \mathrm{CI}$ & $P$ values \\
\hline \multicolumn{6}{|c|}{ Hospitalization through ED } \\
\hline Homeless & 1218 & 94.2 & 1.09 & \multirow[t]{2}{*}{$1.04,1.14$} & \multirow[t]{2}{*}{$<0.001$} \\
\hline Non-homeless & 16,162 & 86.6 & Reference & & \\
\hline \multicolumn{6}{|c|}{ Mechanical ventilation use, including non-invasive and invasive } \\
\hline Homeless & 172 & 13.3 & 1.58 & \multirow[t]{2}{*}{$1.03,2.43$} & \multirow[t]{2}{*}{0.04} \\
\hline Non-homeless & 1884 & 10.1 & Reference & & \\
\hline \multicolumn{6}{|l|}{ In-hospital death } \\
\hline Homeless & 18 & 1.4 & 1.03 & \multirow[t]{2}{*}{$0.63,1.69$} & \multirow[t]{2}{*}{0.91} \\
\hline Non-homeless & 465 & 2.5 & Reference & & \\
\hline
\end{tabular}

CI confidence interval. A Poisson regression was applied with the hospital-level clustered standard errors. Adjusted models included age [5-year intervals], sex, racelethnicity [non-Hispanic White, non-Hispanic Black, Hispanic, and others], primary payer [Medicare, Medicaid, private insurance, self-pay, and others], and indicator variables for 29 comorbidities included in Elixhauser Comorbidity Index. Of the total 20,078 inpatients, we analyzed 19,951 (99.4\%) inpatients without missing key variables. Two-tailed P values below 0.05 were interpreted as statistically significant. We used Stata version 15 (StataCorp., 2017) 
Role of the Funder/Sponsor: None.

Atsushi Miyawaki, $M D, P h D^{1}$

Kohei Hasegawa, $\mathrm{MD}, \mathrm{MPH}^{2,3}$

Yusuke Tsugawa, MD, $\mathrm{MPH}, \mathrm{PhD}^{4,5}$

${ }^{1}$ Department of Public Health, Graduate School of Medicine, The University of Tokyo,

Tokyo, Japan

${ }^{2}$ Department of Emergency Medicine, Massachusetts General Hospital,

Boston, MA, USA

${ }^{3}$ Harvard Medical School,

Boston, MA, USA

${ }^{4}$ Division of General Internal Medicine and Health Services Research, UCLA David Geffen School of Medicine,

Los Angeles, CA, USA

${ }^{5}$ Department of Health Policy and Management, UCLA Fielding School of Public Health,

Los Angeles, CA, USA

Corresponding Author: Atsushi Miyawaki, MD, PhD; Department of Public Health, Graduate School of Medicine, The University of Tokyo Tokyo, Japan (e-mail: amiyawaki-tky@umin.ac.jp).

Author Contributions Dr. Miyawaki had full access to the data in the study and takes responsibility for the accuracy and integrity of the data and its analyses.

Study concept and design: All authors.

Acquisition, analysis, or interpretation of data: All authors.

Drafting of the manuscript: All authors.

Critical revision of the manuscript for important intellectual content: All authors.
Statistical analysis: All authors.

Administrative, technical, or material support: Tsugawa.

Study supervision: Tsugawa.

Funding Information This study was funded by St. Luke's International University, Tokyo, Japan.

\section{Compliance with Ethical Standards:}

The study was approved by the UCLA Institutional Review Board.

Conflict of Interest: The authors declare that they do not have a conflict of interest.

\section{REFERENCES}

1. US Department of Housing and Urban Development. CoC Homeless Populations and Subpopulations Reports. https://www.hudexchange. info/programs/coc/coc-homeless-populations-and-subpopulations-reports/. Accessed Mar 27, 2020.

2. United States Interagency Council on Homelessness. Opening Doors. Federal Strategic Plan to Prevent and End Homelessness, as Amended in 2015.; 2015.

3. Raoult D, Foucault C, Brouqui P. Infections in the homeless. Lancet Infect Dis. 2001;1(2):77-84. doi:https://doi.org/10.1016/S1473-3099(01) 00062-7

4. Wadhera RK, Khatana SAM, Choi E, et al. Disparities in care and mortality among homeless adults hospitalized for cardiovascular conditions. JAMA Intern Med. 2019. doi:https://doi.org/10.1001/ jamainternmed.2019.6010

5. US Department of Commerce. US Census Bureau. https://www.census. gov. Accessed Mar 26, 2020.

6. Leung CS, Ho MM, Kiss A, Gundlapalli AV, Hwang SW. Homelessness and the Response to Emerging Infectious Disease Outbreaks: Lessons from SARS. J Urban Health. 2008;85(3):402-410. doi:https://doi.org/10.1007/ s11524-008-9270-2

Publisher's Note: Springer Nature remains neutral with regard to jurisdictional claims in published maps and institutional affiliations. 